\title{
IVIg modulates BCR signaling through CD22 and promotes apoptosis in mature human B lymphocytes
}

\author{
Jean-François Séïté, ${ }^{1}$ Divi Cornec, ${ }^{1,2}$ Yves Renaudineau, ${ }^{1,2}$ Pierre Youinou, ${ }^{1,2}$ Rizgar A. Mageed, ${ }^{3}$ and Sophie Hillion ${ }^{1}$ \\ ${ }^{1}$ EA2216 Immunology and Pathology and Institut Fédératif de Recherche 148, Université de Bretagne Occidentale, Brest, France; ${ }^{2}$ Laboratory of Immunology, \\ Brest University Medical School Hospital, Brest, France; and 3William Harvey Research Institute, Queen Mary School of Medicine, London, United Kingdom
}

\begin{abstract}
Among various mechanisms for interactions with B cells, intravenous immunoglobulin (IVIg) may operate through the insertion of its Fc part into the Fc- $\gamma$ receptor, or the binding of its sialic acid (SA)bearing glycans to the negatively regulating CD22 lectin. It appeared that IVIg reduces $B$ lymphocyte viability in a dose-
\end{abstract}

and time-dependent manner. Furthermore, we show by confocal microscopy that SApositive IgG, but not SA-negative IgG bind to $\mathrm{CD} 22$. This interaction reduces the strength of B-cell receptor-mediated signaling trough down-regulating tyrosine phosphorylation of Lyn and the B-cell linker proteins, and up-regulating phospholipase $\mathrm{C}_{\gamma 2}$ activation. This cascade resulted in a sustained activation of Erk 1/2 and arrest of the cell cycle at the $G_{1}$ phase. These changes may be accounted for the efficacy of IVIg in autoimmune diseases. (Blood. 2010;116(10): 1698-1704)

\section{Introduction}

Although intravenous immunoglobulin (IVIg) is widely used to treat idiopathic thrombocytopenic purpura, Kawasaki disease, systemic lupus erythrematosus and Guillain-Barré syndrome, ${ }^{1}$ the mechanisms by which immune functions are influenced by such preparations remain to be delineated. ${ }^{2}$ Because B lymphocytes play a central role in the immunopathologic processes that cause these diseases, several studies have suggested that B cells are the target cells for the beneficial effect of IVIg. For this hypothesis to apply, IVIg would modulate a broad range of B lymphocyte functions including activation, proliferation, ${ }^{3}$ and survival. ${ }^{4}$ In other words, IVIg would interfere with the expression of genes and, potentially, the functions of proteins involved in cell growth and death.

The interaction between IVIg and B lymphocytes could thus modulate intracellular signaling resulting from the engagement of the B-cell antigen (Ag) receptor (BCR). The earliest signaling events of the ensuing cascade involves activation of the Src-family protein-tyrosine kinase (PTK) Lyn. ${ }^{5}$ Activated Lyn phosphorylates immunoreceptor tyrosine-based activation motifs (ITAMs) on the cytoplasmic domain of the membrane $\alpha$ and $\beta$ proteins (CD79 $\alpha$ and $\mathrm{CD} 79 \beta$ ). Once phosphorylated, $\mathrm{CD} 79 \alpha$ and $\mathrm{CD} 79 \beta$ recruit another PTK protein, Syk. Syk associates with phospho-ITAMs via its tandem Src-homology 2 (SH2) domains, is phosphorylated, and in turn, phosphorylates the adaptor protein B-cell linker protein (BLNK). The adaptor thus acquires the capacity to bind to and activate Bruton's tyrosine kinase (Btk). This sequence of signaling events leads to the activation of phospholipase C $\gamma 2$ (PLC $\gamma 2$ ), hydrolysis of phosphatidylinisitol-4,5-bisphosphate and the production of inositol-1,4,5-trisphosphate.

As a result, released intracellular calcium synergizes with other signals to induce gene transcription. ${ }^{6}$ This promotion implies the recruitment of several transcription factors (TFs) that influence B-cell proliferation, differentiation, cytokine production, and apoptosis. ${ }^{7,8}$ The outcome of BCR engagement is variable, depending on the status of the
B cell and commitment of coreceptors. The latter dictate the ultimate response and associate positive regulators (such as CD19 and CD21) and negative regulators (such as CD22 and CD32) and these ultimately determine the outcome of B-cell responses. Under normal conditions, both groups of proteins are expressed at varying levels on the surface of all B lymphocytes and their engagement contributes to setting the threshold of B-cell activation. This is often achieved by increasing or decreasing tyrosine phosphorylation, thereby enhancing or reducing signal transduction. ${ }^{9}$

CD22 conveys the $\mathrm{SH} 2$ domain-containing phosphatase 1 (SHP-1) over to the BCR through the immunoreceptor tyrosinebased inhibition motifs (ITIMs) located in its cytoplasmic domain. ${ }^{10}$ This membrane protein belongs to the sialic acid (SA)binding Ig-like lectin (Siglec) superfamily, with 7 Ig-like extracellular domains and an amino-terminal Ig domain. CD22 is unique in that its amino terminal domain recognizes the galactoselinked SA $\alpha 2-6$ sequence that is typically found on N-linked glycans of glycoproteins widely expressed on the surface of various cell types. Known ligands for CD22 include polysaccharides attached to antibodies (Abs), ${ }^{11}$ but the intimate nature of the binding of CD22 to endogenous glycoproteins in a cis manner remains to be determined. In contrast, there is good evidence that CD22 binding by exogenous cross-linkers affects B-cell responses. ${ }^{12}$ Whether different ligands produce different effects on $\mathrm{B}$-cell responses is unclear, yet the ligand-binding domain of CD22 most notably its $\alpha 2-6-$ linked SA-binding domain is needed for CD22 to inhibit BCR signaling. ${ }^{13,14}$

To gain insights into how IVIg modulates immune responses, we explored the capacity of IVIg to modulate BCR signaling and thus influence the fate of B cells. Our results suggest that SAcontaining IgG within IVIg peparations bind to CD22 on B cells. This binding modulates B-cell functions, and could therefore contribute to the therapeutic efficacy of IVIg. 


\section{Methods}

\section{B-cell isolation and culture}

Blood samples were collected from healthy volunteers, and tonsils from children undergoing routine tonsillectomy. All volunteers and parents of children gave informed consent in accordance with the Declaration of Helsinki, and the study was approved by the Institutional Review Board at the Brest University Medical School Hospital. Peripheral blood mononuclear cells were separated on Ficoll-Hypaque, T lymphocytes rosetted out, and B-cell-enriched preparations collected. All monoclonal Abs (mAbs) were from Beckman-Coulter unless otherwise specified. Fluorescein isothiocyanate (FITC)-conjugated anti-CD19 and phycoerythrin (PE)conjugated anti-CD5 mAbs were combined to ensure the sample purity by fluorescence-activated cell sorting (FACS) analyses. There were more than 98\% B lymphocytes in all preparations.

Human Ramos lymphoma line B cells were purchased from ATCC. B cells were cultured in RPMI- 1640 medium containing $10 \%$ fetal calf serum and supplemented with $2 \mathrm{mM}$ L-glutamine, $200 \mathrm{U} / \mathrm{mL}$ penicillin and $1 \mu \mathrm{g} / \mathrm{mL}$ streptomycin.

\section{Assessment of apoptosis}

To assess their apoptosis, $2 \times 10^{5} \mathrm{~B}$ cells in RPMI-1640 were incubated for 24 hours with increasing amount of IVIg (LFB). Cells were then stained with FITC-annexin $\mathrm{V}(\mathrm{AV})$ and propidium iodide (PI) according to Beckman Coulter's instructions. Briefly, B cells were suspended in $100 \mu \mathrm{L}$ phosphate-buffered saline (PBS) buffer and incubated for 10 minutes at $4^{\circ} \mathrm{C}$ with $4 \mu \mathrm{L}$ PI, and $1 \mu \mathrm{L}$ FITC-AV. In the FACS analyses, cells in the early stage of apoptosis were positive for FITC-AV and negative for PI. They were enumerated, and their mean fluorescence intensities (MFIs) recorded.

\section{IVIg preparation}

IVIg was dissolved in serum-free RPMI-1640 medium to a concentration of $100 \mathrm{mg} / \mathrm{mL}$, and prepared for conjugation with FITC by dialysis of IVIg in carbonate-bicarbonate buffer, $\mathrm{pH}$ 9.5. Fluorescein-5-thiosemicarbazide (Molecular Probes) was then added with $100 \mu \mathrm{g}$ in dimethylsulfoxyde for each $1 \mathrm{mg}$ of IVIg for 1 hour at $37^{\circ} \mathrm{C}$. Excess FITC was removed by extensive dialysis against PBS. This preparation was assessed in an UV2 spectrophotometer (Unicam) by reading the absorbance at $280 \mathrm{~nm}$ and $495 \mathrm{~nm}$, and by expressing the fluorescence as [(absorbance $280 \mathrm{~nm}$ ) -0.31 (absorbance $495 \mathrm{~nm})] / 1.4$. An IVIg-devoid preparation was examined in parallel to guarantee that the fluorescence came from conjugated IVIg, and not from dissociated FITC.

SA-IgG was purified from the bulk of IVIg preparations by lectinaffinity chromatography with a Sambucus nigra agglutinin (SNA) agarose column (Vector Laboratories). Enrichment of the eluate was verified by Western blotting (WB) with biotinylated SNA at $4 \mu \mathrm{g} / \mathrm{mL}$ and horseradish peroxidase (HRP)-conjugated Streptavidin.

For preparing Fab and Fc fragment, IVIg preparations were digested with $2 \mathrm{mg} / \mathrm{mL}$ papain in $0.04 \mathrm{M}$ cystein-PBS for 6 hours at $37^{\circ} \mathrm{C}$. The resulting $\mathrm{Fab}$ and $\mathrm{Fc}$ fragments were separated from the undigested IVIg using a Sepharose S300 column and the Fc fragments were further separated from the Fab fragments on a protein $\mathrm{G}$ column. All purification steps were carried out using a fast protein liquid chromatography system (Pharmacia). Purity of the fragments was assessed by WB using Abs specific either for Fab of for Fc.

\section{Analysis of phosphorylation by FACS}

Phosphorylation assays were carried out using PE-conjugated mouse anti-phospho-BLNK (Phosphotyrosine PY 84; BD Biosciences), PEconjugated mouse anti-phospho-PLC $\gamma 2$ (PY759) mAbs (BD Biosciences), rabbit anti-phospho-CD19 (PY531) Ab, rabbit anti-phospho-Lyn (PY396) and rabbit anti-phospho-CD22 (PY822) Abs (all from Abcam) developed by FITC donkey anti-rabbit Ab (Jackson ImmunoResearch Laboratories). For FACS analysis of phosphorylation, the cells were either unstimulated or stimulated with $20 \mu \mathrm{g} / \mathrm{mL}$ anti-IgM-coated onto beads in the presence, or absence of $20 \mathrm{mg} / \mathrm{mL}$ IVIg for 20 minutes at $4^{\circ} \mathrm{C}$, and then for 3 minutes at $37^{\circ} \mathrm{C}$. In some experiments, $1 \mathrm{mg} / \mathrm{mL}$ sialylated IVIg (IVIg SA+), or $20 \mathrm{mg} / \mathrm{mL}$ nonsialylated IgG (IVIg SA-) was added. Activated cells were fixed and permeabilized by incubation for 30 minutes in $70 \%$ methanol before staining. Data were analyzed using EXPO32 program. Changes in phosphorylation expressed in folds increase and decrease were calculated in cells stimulated in the presence or absence of IVIg.

\section{Confocal microscopy}

To evaluate its relationships with CD22, $20 \mathrm{mg} / \mathrm{mL}$ of IVIg was associated with $5 \times 10^{5} \mathrm{~B}$ lymphocytes. Interactions were revealed using goat FITC-conjugated $\mathrm{Fab}_{2}{ }_{2}$ anti-human IgG Ab (Jackson ImmunoResearch Laboratories), and rabbit anti-CD22 (Abcam), wich was revealed with tetrarhodamine isothiocyanate (TRITC)-conjugated donkey anti-rabbit Ig $\mathrm{Ab}$ (Jackson ImmunoResearch Laboratories). In some experiments, $1 \mathrm{mg} /$ $\mathrm{mL}$ of purified IVIg SA + or $20 \mathrm{mg} / \mathrm{mL}$ of the IVIg SA - fraction was added to $5 \times 10^{5}$ tonsil $\mathrm{B}$ cells, and binding revealed as before. After a 1-hour incubation at $4^{\circ} \mathrm{C}$, B lymphocytes were fixed with $4 \%$ filtered p-formaldehyde solution, cytospined and visualized on a Leica TCS laser scanning confocal microscope at $100 \times$ magnification. Slides were mounted with Vectashield from Vector Laboratories. Acquisition was made with TCS NT Leica software.

\section{Immunoprecipitation}

B cells were incubated with $20 \mu \mathrm{g} / \mathrm{mL}$ anti-IgM Ab-coated beads and/or $20 \mathrm{mg} / \mathrm{mL}$ IVIg for 20 minutes at $4^{\circ} \mathrm{C}$ before activation at $37^{\circ} \mathrm{C}$ for 3 minutes. The cells were then lysed in $50 \mathrm{mM}$ Tris (hydroxymethyl) aminomethane, $150 \mathrm{mM} \mathrm{NaCl}, 5 \mathrm{mM}$ sodium fluoride, $2 \mathrm{mM}$ ethylenediaminetetraacetic acid, $40 \mu \mathrm{M}$ sodium orthovanadate, protease inhibitor cocktail and $0.2 \%$ Brij-96 (all from Sigma-Aldrich) for 1 hour at $4{ }^{\circ} \mathrm{C}$. After centrifugation, supernatants were collected and pre-cleared with $50 \mu \mathrm{L}$ protein G-coated Sepharose beads for 30 minutes at $4^{\circ} \mathrm{C}$. The lysates were incubated with $10 \mu \mathrm{g} / \mathrm{mL}$ rabbit anti-CD22 Ab, or rabbit anti-PY476-SHP-1 Ab and $100 \mu \mathrm{L}$ protein G-coated Sepharose beads overnight at $4{ }^{\circ} \mathrm{C}$. The beads were washed 5 times with lysis buffer and bound proteins in the immunoprecipitates eluted with $80 \mu \mathrm{L}$ of warm reducing loading buffer containing $0.125 \mathrm{mM}$ Tris, $10 \%$ glycerol, $4 \%$ SDS and $1.8 \% \beta$-mercaptoethanol, and separated on $7 \%$ polyacrylamide gel electrophoresis (PAGE). WB was carried out with the same anti-CD22 and anti-SHP-1 Abs. Bindings of the Abs was revealed with HRP-conjugated anti-rabbit Ab (Amersham) and enhanced chemiluminescence (ECL) detection kit.

\section{Western blotting}

Lysates were prepared as described above and analyzed on $7 \%$ to $13 \%$ SDS-PAGE. Separated proteins were transferred to a polyvinylidene difluoride membrane, and quenched with 5\% nonfat milk in PBS or with $5 \%$ of BSA for 1 hour at room temperature and then incubated with primary $\mathrm{Ab}$ for 1 to 6 hours, depending on the Ab. Rabbit anti-caspase 9 and anti-caspase 3 Abs were from Cell Signaling Technology. Rabbit antiCDK2, mouse anti-E2F, anti-phosphoP38 (PT180+PY182), anti-phosphoErk1/Erk2, anti-p27, and anti-p85 of phosphoinositide 3-kinase Abs were from Abcam. The anti-poly ADP-ribose polymerase (PARP) was Ab from Roche Applied Science and HRP-conjugated sheep anti-mouse as well as HRP-conjugated donkey anti-rabbit Abs were from GE Healthcare. These Abs were used at 1 in 40000 dilution in $1 \%$ nonfat milk protein containing 0.05\% Tween in PBS. Bound Abs were revealed using the ECL kit.

\section{Statistical analyses}

Analysis of variance and 2-tailed paired Student $t$ test were used to determine the significance of difference between sample means. Significance refers to difference from the controls unless otherwise indicated. 

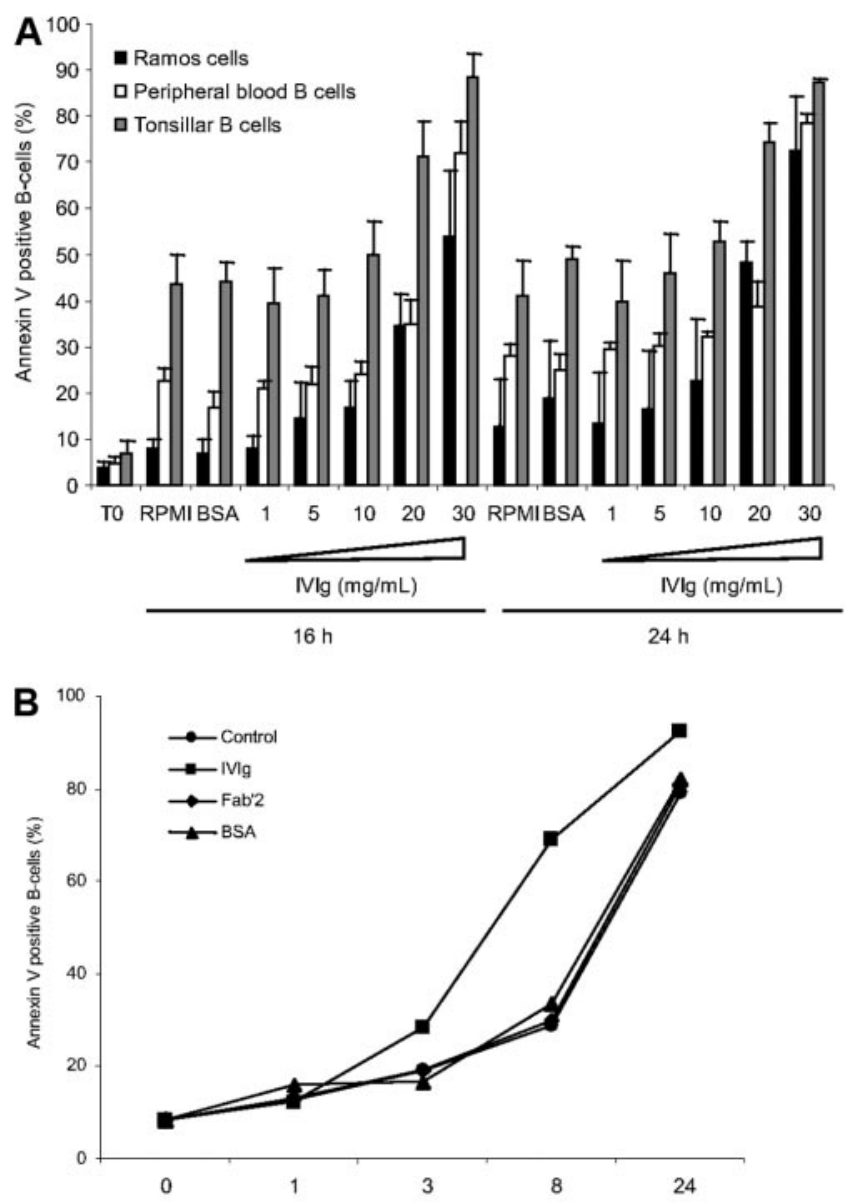

hours

Figure 1. Reduced B-cell viability in the presence of IVIg. (A) Blood and tonsillar $B$ cells and lymphoma cell line B cells $\left(2 \times 10^{5}\right)$ were incubated for 24 hours with increasing concentrations of IVIg. Cell viability was assessed by FITC-AV uptake. BSA was used at $20 \mathrm{mg} / \mathrm{mL}$ as a control for the effect of high protein content. (B) Tonsillar B cells were cultured in the presence of $20 \mathrm{mg}$ of IVIg ( $\square)$, IgG F(ab') $(\bullet), \operatorname{BSA}(\boldsymbol{\Delta})$, or in medium alone $(\bullet)$ for the indicated periods. The figure depicts the mean \pm SD values from 5 independent experiments. Statistical analysis was performed using the 2-tailed paired Student $t$ test. ${ }^{\star} P<.05$ vs cells cultured in medium alone.

\section{Results}

\section{IVIg induces B-cell apoptosis through a selective pathway}

We first examined the influence of IVIg on B-cell survival. FITC-AV/IP staining of blood and tonsillar primary B cells and lymphoma B-cell line cells revealed that these cells underwent apoptosis in a dose-dependent and time-related manner in the presence of IVIg (Figure 1A and supplemental Figure 1, available on the Blood Web site; see the Supplemental Materials link at the top of the online article). IVIg at $20 \mathrm{mg} / \mathrm{mL}$ significantly reduced cell viability at 16 to 24 hours $(P<.005)$. Thus, the basal rate of apoptosis at 16 and 24 hours were significantly enhanced by the presence of IVIg. Intriguingly, B lymphocytes from blood and Ramos cells were somewhat resistant to spontaneous and IVIginduced death, compared with tonsillar B cells.

To ensure that IVIg was the genuine killing agent, this preparation was dialyzed against RPMI 1640 to exclude that stabilizers were responsible for cell death. We repeated the experiments using $\mathrm{Fab}_{2}$ fragments of IVIg instead of the whole molecules. Culturing dialyzed IVIg preparation with tonsillar
B cells showed similar kinetics, thus excluding a role for stabilizers. In addition, significant differences in the uptake of $\mathrm{AV}$ conjugate by the $\mathrm{B}$ cells was seen when the cells were cultured in the presence of the complete Ig comparing with their $\mathrm{F}\left(\mathrm{ab}^{\prime}\right)_{2}$ fractions at 8 hours (Figure 1B). Next, the induction of apoptosis was assessed with high protein concentration by comparing the rate of cell death in the presence of IVIg with an equimolar concentration of bovine serum albumin (BSA). The apoptosis rate in the presence of BSA was identical to spontaneous apoptosis and significantly less than with $\operatorname{IVIg}(P<.01)$.

\section{The binding of IVIg to B cells is mediated by interaction with CD22}

Given that no direct interaction between IVIg and B cells has hitherto been proven, we labeled IVIg with FITC to obtain direct evidence for its binding to B cells. The IVIg bound Ramos cells in a dose-dependent manner (Figure 2A), until the cells were saturated, thus, confirming specificity of the binding. To identify the potential molecule through which IVIg bind B cells, we analyzed the interactions between IVIg and B cells obtained from different sources. This experiment was carried out based on the hypothesis that different B-cell populations would have different expression levels of the molecule(s) to which the IVIg binds. This experiment revealed that, indeed, the IVIg bound more to Ramos cells than to cells from tonsils and blood (Figure 2B). IVIg could thus have a selective effect on a subpopulation of B cells.

To gain insight into how IVIg modulate B-cell functions, we examined the capacity of IVIg to bind potential membrane proteins on the B cells. B cells express several molecules that have potential to modulate B-cell functions upon interaction with their specific ligands and receptors. CD22, a member of the SA binding Ig Siglec family, is one such key molecule. It is a B cell-specific glycoprotein that associates with the BCR. The Fc parts of IVIg have a Siglec N-linked glycan covalently linked with a core containing $\mathrm{N}$-acetylglucosamine and mannose. Further modification of this core is observed in the IgG pool of which 5\% terminate with SA.

Confocal microscopy analyses served to address the issue of CD22 as a ligand for IVIg. The use of FITC-conjugated IVIg confirmed that within the IVIg preparation, some IgG bind to the

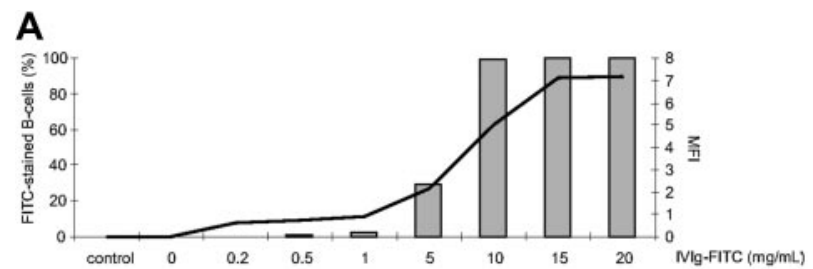

B

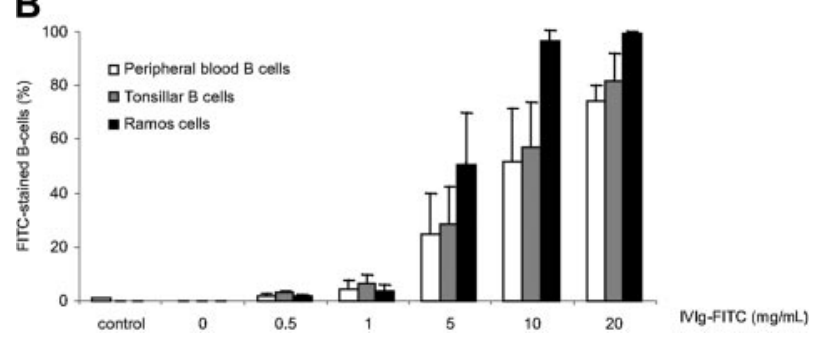

Figure 2. IVIg binds to B-cell surface in a dose-dependent manner. (A) Ramos $B$ cells were incubated with a range of FITC-labeled IVIg for 1 hour at $4^{\circ} \mathrm{C}$. Binding of IVIg was determined by FACS and MFIs measured at each point of FITC-IVIg binding. Unconjugated FITC was used as the negative control. (B) Blood B cells (open bars), tonsillar B cells (gray bars) and Ramos B cells (closed bars) were incubated with increasing concentrations of IVIg-FITC and binding measured by FACS. 
surface of B cells (Figure 3A). This was contrasted with the binding of aggregated $\mathrm{IgG}$, used as positive control and goat anti-human IgG as a negative control. The pattern of IVIg binding to the B cells membrane was somewhat similar to that obtained for the binding of aggregated IgG. However, the binding of aggregated IgG show larger green fluorescence lumps than seen for the IVIg, which appears to spread thinner on the surface. Overlay of FITC-conjugated IVIg and TRITC-conjugated CD22 shown as yellow clearly indicated that IVIg and CD22 colocalized on the B-cell surface (Figure 3B). To directly show that the interaction between CD22 and IVIg involved SA on IgG, IVIg SA+ and IVIg $\mathrm{SA}-$ fraction were obtained and their purity verified by WB

A

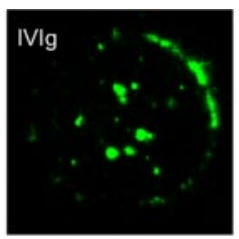

B

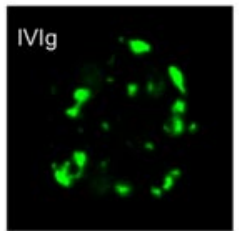

C
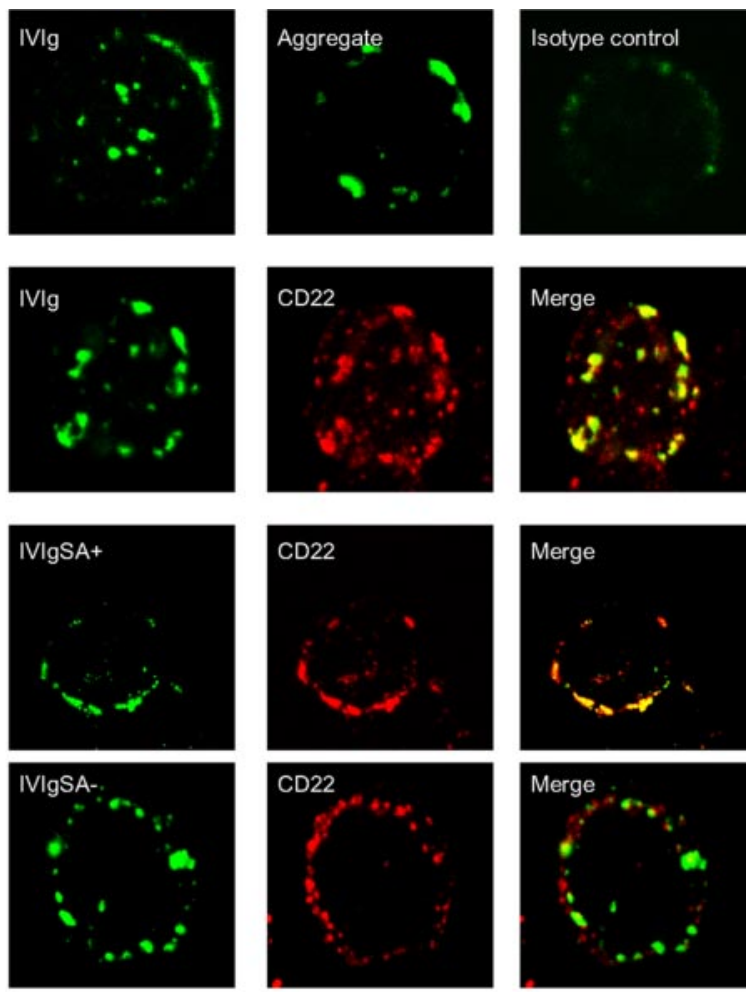

D

Sambucus nigra Lectin blot

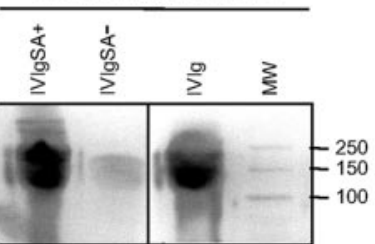

E

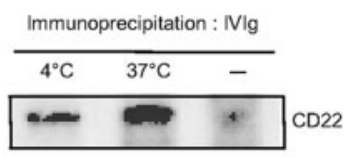

Figure 3. IVIg binds to CD22 on the surface of B cells. (A) Tonsillar B cells were incubated with IVIg (left panel), or with aggregate IgG (middle panel). The binding of IgG was revealed using FITC-conjugated goat Fab'2 anti-human IgG. The right panel shows binding of the secondary $\mathrm{Ab}$ alone with the TRITC-conjugated donkey anti-rabbit IgAb, used as negative control. (B) Tonsillar B cells were stained with rabbit anti-CD22 followed by TRITC conjugated anti-rabbit Ab. After several washes, the cells were incubated with IVIg and and followed by FITC-conjugated anti-human IgG $F\left(a b^{\prime}\right)_{2}$. (C) Sialylated IVIg (IVIg SA + ) and asialylated (IVIg SA-) fractions were used to stain B cells followed by FITC-conjugated $F\left(a^{\prime}\right)_{2}$ anti-human IgG antibody. $B$ cells were also stained with anti-CD22. (D) IVIg was separated according to their SA content using SNA affinity chromatography. Purity of the fractions was assessed by lectin blotting. Staining for CD22 was followed by secondary HRP-conjugated Ab, and the blots developed using the ECL system. (E) Tonsillar B cells were incubated with IVIg before warming the cells up at $37^{\circ} \mathrm{C}$ (middle lane) or examined without warming (left lane). Control for warming up at $37^{\circ} \mathrm{C}$ without IVIg is also shown (right lane) is shown. After the incubation, cell lysates were prepared and immunoprecipates with protein $\mathrm{G}$ Sepharose beads and analyzed by WB using anti-human CD22 $\mathrm{Ab}$ followed by secondary HRP conjugated $\mathrm{Ab}$. The blots were developed using the ECL system.
(Figure 3D). IVIg and IVIgSA + fraction, but not IVIg SA - bound SNA. Thus, only the IVIg SA+ fraction colocalized with CD22 (Figure 3C upper panel), although some in the IVIg SA- fraction also bound to the B-cell membrane (Figure 3C lower panel).

Binding of the IVIg fraction to CD22 was further confirmed by immunoprecipitation. B cells were incubated with the IVIg preparations for 20 minutes at $4{ }^{\circ} \mathrm{C}$ and for 5 minutes at $37^{\circ} \mathrm{C}$, lysed and bound IgG-CD22 immunoprecipitated. Within the IVIg, IgG was associated with CD22 on the WB (Figure 3E). Interestingly, these experiments showed that the optimal binding of the IVIg preparation with CD22 occurred when the cell-IVIg mixtures were maintained at $37^{\circ} \mathrm{C}$. These experiments provide evidence that the binding of $\mathrm{IgG}$ to $\mathrm{CD} 22$ is optimal at a physiologic temperature.

\section{Effect of IVIg on BCR-mediated signaling involves CD22}

To assess the likely effects of IVIg binding to B cells on BCRmediated signaling, we examined the strength of anti-IgMgenerated signaling. Although the exact dynamics of signaling events that translate BCR engagement to cell responses are not fully established, modulation of the level of intracellular calcium level is considered a reliable measure.

In addition, we assessed the phosphorylation status of several key molecules associated with BCR-mediated signaling by FACS. This technique allows the quantification of PY in individual subpopulations of cells, and reduces the effects of manipulation on measurement of B cells. Analyses were performed on gated viable cells. After 3 minutes of stimulation with anti-IgM, 22\% of tonsillar B cells showed elevated levels of phosphorylated CD22 at its ITIMs in the absence of IVIg (Figure 4A). The percentage phosphorylated of CD22 in B-cells increased from 22 in cells cultured without IVIg to 58 of total CD22 in the presence of IVIg. In contrast, tyrosine phosporylation of CD19 diminished. The analysis also showed decreased phosphorylation of Lyn, and of BLNK at Y84, which is one of the 3 tyrosine residues involved in recruiting PLC $\gamma 2 .{ }^{15}$ Indeed, there was a significant reduction $(P<.001)$ in PLC $\gamma 2$ phosphorylation at Y759 in the presence of IVIg. No changes in the phosphorylation level of the signaling proteins were seen in unstimulated $\mathrm{B}$ cells in the absence, or presence of either total IVIg or its fractions (Figure 4A). However, when $\mathrm{B}$ cells were stimulated with anti-IgM in the presence of either $20 \mathrm{mg} / \mathrm{ML}$ total IVIg or with $1 \mathrm{mg} / \mathrm{mL}$ IVIg SA+ there was a significant elevation in the level of CD22 phosphorylation and reduction in CD19, Lyn, BLNK and PLC $\gamma 2$ phosphorylation (Figure 4B). The IVIg SA - fraction, in contrast, did not alter the level of phosphorylation of these proteins even at $20 \mathrm{mg} / \mathrm{mL}$. All the noted down-regulation of phosphorylation strongly indicated an effective modulation of the calcium pathway.

To confirm that the reduction in the calcium signaling pathway was due to CD22, we immunoprecipitated CD22 and measured associated SHP-1. As a result of hypo-phosphorylation of CD19, Lyn, BLNK and PLC $\gamma 2$, SHP-1 was more recruited by adherence of IVIg to CD22 (Figure 5A). The constitutive association between CD22 and SHP-1 was slightly increased upon BCR cross-linking but significantly so in the presence of IVIg (Figure 5B). The ability of CD22 to modulate B-cell responses by interacting in trans with glycosylated Ags has previously been demonstrated. ${ }^{16}$ Here, we show that high concentrations of IVIg could act in a similar manner and reduce the strength of BCR-mediated signaling. This occurs through the CD22-SHP-1 inhibitory pathway and by downregulating calcium pathway activation, that determine the fate of B cells. 
A

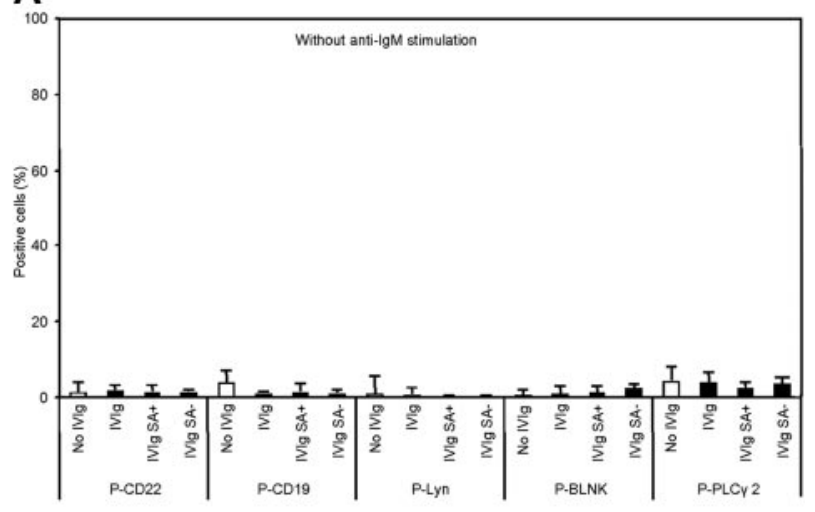

B

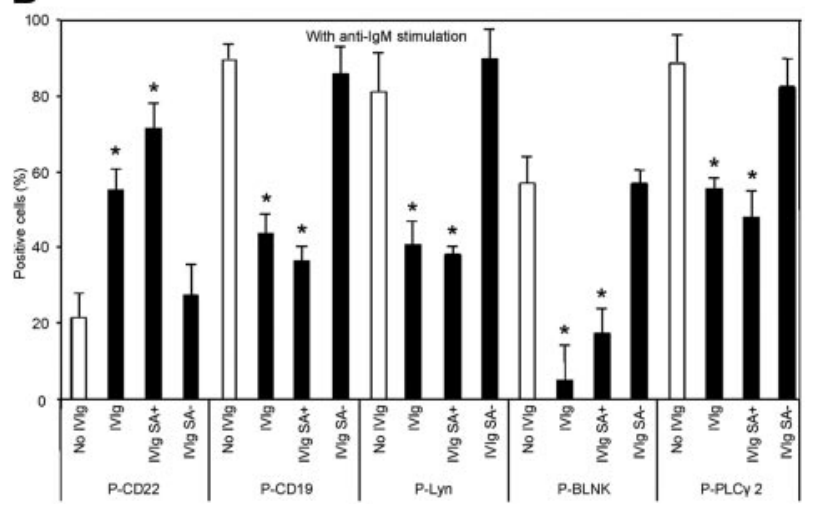

Figure 4. IVIg modulate BCR-associated signaling pathways. Phosphorylation levels of CD22, CD19, Lyn, BLNK and PLC $\gamma 2$ were determined by FACS. (A) Tonsillar B-cell subpopulations were incubated with or without $20 \mathrm{mg} / \mathrm{mL}$ IVIg or $1 \mathrm{mg} / \mathrm{mL} \mathrm{IVIg}$ $\mathrm{SA}+$ or $20 \mathrm{mg} / \mathrm{mL}$ IVIg SA - for 20 minutes at $4^{\circ} \mathrm{C}$ and warmed up for 3 minutes at $37^{\circ} \mathrm{C}$ without any stimulation. B cells were subsequently fixed with $\mathrm{p}$-formaldehyde, permeabilized and then stained with rabbit anti-phospho-CD22, anti-phosphoCD19, and anti-phospho-Lyn and developed by FITC-donkey anti-rabbit Ig Ab, and with PE-conjugated anti-phospho-BLNK and anti-phospho-PLC $\gamma 2$. (B) Tonsillar B cells were stimulated with anti-IgM Ab-coated beads for 3 minutes at $37^{\circ} \mathrm{C}$ in the presence or absence of $20 \mathrm{mg} / \mathrm{mL}$ IVIG, $1 \mathrm{mg} / \mathrm{mL}$ IVIg SA+ or $20 \mathrm{mg} / \mathrm{mL}$ IVIg SA - for 20 minutes at $4^{\circ} \mathrm{C}$. Activated $B$ cells were stained with anti-phospho-CD22, antiphospho-CD19, anti-phospho-Lyn, anti-phospho-BLNK and anti-phospho-PLC $\gamma 2$ with the same protocol as before. The results represent mean percentages \pm SD of 3 independent experiments. Statistical analyses were performed using the 2-tailed paired Student $t$ test. ${ }^{*} P<.001$ vs cells incubated with no IVIg.

\section{IVIg promotes apoptosis through sustained activation of Erk 1/2 and cell-cycle arrest}

Erk1/2 phosphorylation results from BCR signaling, and influences the behavior of B-cells. ${ }^{17}$ As IVIg binding influences BCR signaling, we next assessed the effect of this interaction on Erk1/2 phosphorylation. For this purpose, we studied tonsillar B cells in presence of IVIg, with and without BCR engagement. Treatment with IVIg for 30 seconds induced Erk1/2 phosphorylation sustained after 2 minutes (Figure 6A). Further study of the impact of IVIg binding on other MAPK kinases showed that IVIg decreased p85 PI3K level but increased p38 phosphorylation (Figure 6B). Erk1/2 thus has a dual role in regulating cell proliferation and cell cycle arrest, so that the outcome of altered phosphorylation of these proteins depends on several factors including duration of the modulating stimulus.

To determine the consequences of these changes in phosphorylation on the fate of B cells, we studied the level of several proteins involved in the cell cycle. Because IVIg promoted apoptosis, we examined the effect of IVIg binding on proteins regulating the $G_{1}$ phase of the cell cycle. Previous studies have highlighted a critical

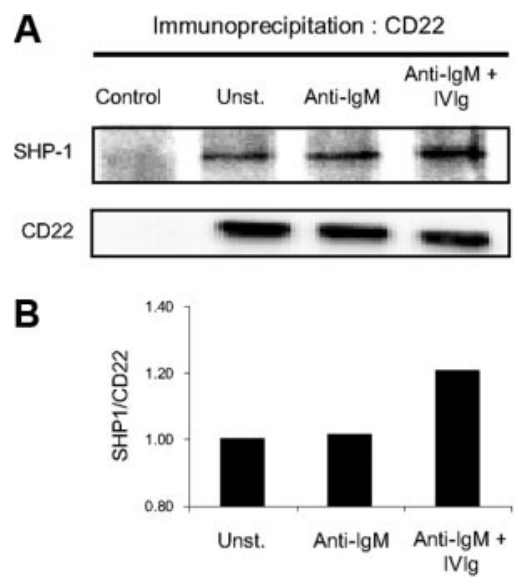

Figure 5. IVIg binding to CD22 enhances SHP-1 recruitment. (A) Tonsillar B cells were incubated with anti-lgM-coated beads in the presence or absence of IVIg for 20 minutes at $4^{\circ} \mathrm{C}$ and 3 minutes at $37^{\circ} \mathrm{C}$ before lysing the cells. Lysates were incubated with anti-CD22 and protein G-coated Sepharose beads and immunoprecipitates analyzed by WB using anti-CD22 or anti-SHP-1 Ab. Nonstimulated B cells (Unst.) are shown as control. (B) The degree of coprecipitation is presented as a ratio of intensity of SHP-1 band/intensity of CD22 band.

role for $\mathrm{p} 27 / \mathrm{Kip} 1$ in cell cycle progression though the $\mathrm{G}_{0}-\mathrm{G}_{1}$ phase, ${ }^{18}$ as we also confirmed in another model. ${ }^{19}$ Treatment of $\mathrm{B}$ cells with IVIg resulted in a significant increase in the level of p27/Kip1 with and without BCR engagement (Figure 7A). Semiquantitative measurements, based on band density showed a 2-fold increase in the level of p27/Kip1 expression. In contrast, a 24-hour treatment of B cells with IVIg abrogated cyclin-dependent kinase $(\mathrm{CDK}) 2$ expression. This reduction was exaggerated by BCR engagement, in line with up-regulation of its inhibitor p27/Kip1.

Because down-regulation of CDK2 inhibits the E2F-1 growth promoting agent, ${ }^{20}$ we therefore evaluated the expression of the latter TF. A 24-hour BCR engagement in the presence of IVIg resulted in a striking reduction in the E2F-1 expression (Figure 7A). Consistent with this data, IVIg induced a $G_{1}$ cell cycle arrest and led to apoptosis. Both processes are controlled by $\mathrm{p} 53$, which governs expression of key pro- and anti-apoptotic molecules such as $\mathrm{Bcl} 2$, which, in turn, regulate activation of caspases. In this respect, treatment of B cells with IVIg and BCR engagement
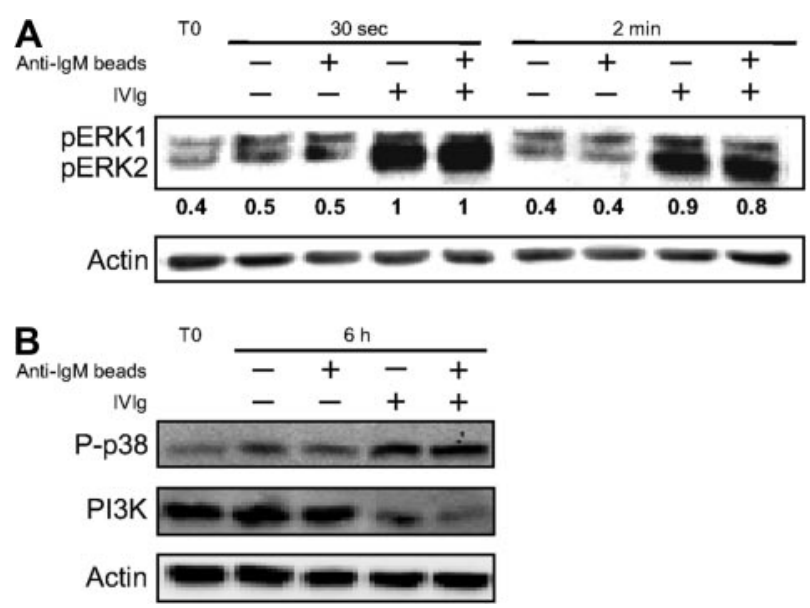

Figure 6. IVIg modulates the MAPK pathway. Tonsillar B cells were incubated with anti-IgM-coated beads in the presence or absence of IVIg for 30 seconds or 2 minutes before lysis. Total cell lysates were prepared for WB using anti-phospho ERK1 and anti-phospho ERK2 (A) or anti-phospho p38 and anti-PI3K (B) Abs. Values indicate relative density of the WB bands normalized to that obtained for $\beta$-actin. Data are representative of 3 separate experiments. 

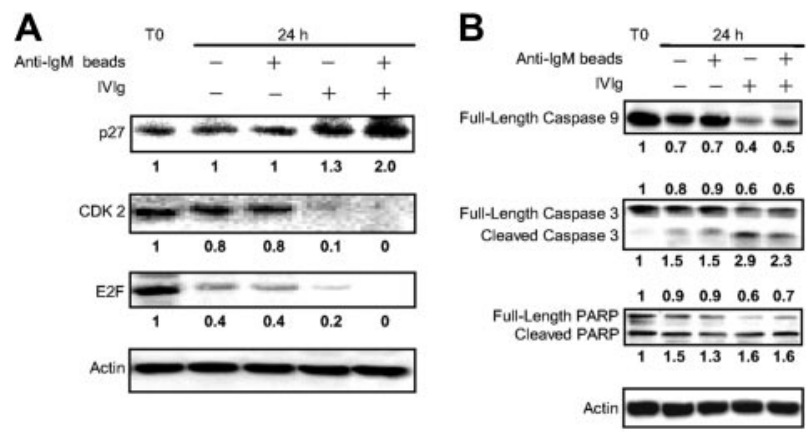

Figure 7. Effect of IVIg on cell cycle protein expression and apoptosis. Tonsillar $B$ cells were incubated with anti-IgM-coated beads in the presence or absence of IVIg for 24 hours before lysis. Total cell lysates were separated and stained in WB with (A) anti-p27, anti-CDK2 and anti-E2F Abs, and (B) anti-caspase 9, anti-caspase 3 , and anti-PARP Abs. Values indicate relative density of the bands normalized to that obtained for $\beta$-actin. Data are representative of 3 independent experiments.

decreased the levels of pro-caspases 3 and 9, and increased that of cleaved PARP (Figure 7B).

All in all, the data generated in this study indicate that IVIg modulates B-cell responses promotes cell cycle arrest and apoptosis by mediating Erk phosphorylation and p38 activation while reducing $\mathrm{PI} 3 \mathrm{~K}$ activation.

\section{Discussion}

An ever-growing array of autoimmune conditions has successfully been treated with IVIg. Although the exact mode of action remains elusive, IVIg modulates the immune system through binding to membrane receptors. B lymphocytes possess some of these receptors. In this study, we provide evidence that CD22 is a key receptor in the beneficial effects of IVIg. Further, we report for the first time that IVIg binds primary human B cells and modulate BCRmediated signaling. Numerous investigators have previously demonstrated that IVIg influences B cells, ${ }^{4,21-23}$ however, none have really identified the pathways through which B-cell fate would be influenced by IVIg. Modulation of Erk activation in B cells by IVIg has been described before ${ }^{21}$ in association with growth inhibition. In this study, we report that IVIg influences the cell cycle and leads to apoptosis. A previous report has proposed that IVIg induces apoptosis though the Fas pathway. Altough our study has identified a different mechanism, we cannot totally exclude apoptosis through the-Fas pathway, because different IVIg preparations can vary as they are from different donors. ${ }^{4}$ Although IVIg has been shown not to affect B-cell viability when stimulated with CD40L, ${ }^{22}$ we observed that B-cell activation through the BCR was critically important, underlying the role of B-cell activation state in IVIginduced apoptosis.

There is good evidence that CD22 interacts in a cis fashion with ligands to control BCR-mediated signaling. However, controversy persists as to the requirement for Ag glycosylation. In this case, IVIg could bind CD22 in trans to influence signaling. In fact, the latter ligands are so ubiquitous that it renders impossible to discriminate the contribution of cis and trans modes of interactions. We reasoned that IVIg SA+ could bind CD22 and interfere with BCR-mediated signaling. Modulation of B-cell activation by IVIg, especially through the calcium pathway, is due to binding to CD22 and recruitment of SHP-1. Together, our results indicate that trans interaction by CD22 suppresses BCR-mediated B-cell activation, as previously proposed. ${ }^{16,24}$ Incubation of IVIg with anti-Ig- activated B cells enhances CD22 phosphorylation, which is necessary for SHP-1 recruitment. ${ }^{14}$ Similar results were obtained using synthetic sialosides to abrogate ligand binding in in vitro and in vivo. ${ }^{25}$ Thus, ligand binding cis and trans modes are required for the inhibitory role of $\mathrm{CD} 22 .{ }^{13}$ In this regard, a recent study has provided evidence for the effect of sialylated antigens on B cells. ${ }^{26}$ These investigators showed that B-cell Ag activation could be modulated by interaction of glycosylated antigen with CD22 leading to the induction of tolerance. The avidity of IVIg-CD22 interaction is a challenge to measure, but probably weaker than the maximum CD22 cross-linking. There are, however, some suggestions that direct interaction between CD22 and Ag could influence the fate of B cells by modulating secondary signals such as Erk. ${ }^{27}$ Furthermore, a link between CD22 engagement and apoptosis has been suggested for B cells in patients with chronic lymphocytic leukemia. ${ }^{28}$ Immunotoxins that bind CD22 would favor cytotoxicity through activating caspase 3 and cleaving PARP. ${ }^{29}$ Our data indicated that a similar outcome of cell death is achieved by IVIg in B cells. However, it remains to be formally demonstrated that cytotoxicity is mediated by IVIg-CD22 interaction in this setting.

Analysis of the potential pathways through which IVIg-CD22 could influences B cells revealed that IVIg enhances Erk1/2 phosphorylation as suggested by others investigators. ${ }^{21,30}$ IVIg also enhanced p38-phosphorylation but down-regulated p85-activation. All these signaling molecules are involved in regulating apoptosis in B cells. ${ }^{31,32}$ The p38 MAPK plays a role in cell cycle progression of $\mathrm{B}$ cells that are exposed to oxidative stress. ${ }^{33}$ In addition, p38 could induce $\mathrm{G}_{1}$ cycle arrest and $\mathrm{Bcl} 2$ phosphorylation leading to its degradation in the proteasome. ${ }^{34}$ p38 and JNK are stressassociated PTKs that regulate apoptosis depending on the cell type and stimulus. ${ }^{35,36}$ Although p38 has been implicated in apoptosis in several systems, the mechanism by which this is brought about is unknown. Hsu and colleagues reported that p38 mediates apoptosis through increased expression of Fas ligand. ${ }^{37}$ Our results showed that IVIg-CD22 interaction enhanced p38 phosphorylation and led to the activation caspase- 3 cleavage, which is consistent with a recent report. ${ }^{38}$

Analysis of potential pathways influenced by IVIg-CD22 interaction indicate that IVIg interferes with BCR signaling and B-cell responses through sustained Erk1/2 phosphorylation, and down-regulation of PI3K activation. The PI3K/Akt pathway (which is essential for survival) constitutes a common response pathway of cells to growth-factor stimulation. Erk1/2 has a dual role in regulating B-cell responses, and is thus required for cell proliferation as well as cell cycle arrest. ${ }^{39,40}$ However, our study could not exclude the possibility that CD22 engagement by IVIg disrupts the MAPK pathway, cell cycle arrest and apoptosis as has been reported for CD22 cross-linking. ${ }^{29,38,41}$

This results in cell cycle arrest at $\mathrm{G}_{1}$ phase, down-regulation of CDK2 and up-regulation of its inhibitor p27, which activates caspase-3. This findings leads to the prediction that other inducible CDKs, such as CDK4 and CDK6, could be down-regulated by IVIg-CD22 interaction. For example, down-regulation of CDK2/6 causes inactivation of $\mathrm{pRb}$, which down-regulates the E2F family of TFs, known as proteins required for $\mathrm{S}$ phase. ${ }^{42}$ Indeed, IVIg reduced E2F-1 protein expression, and thereby related target genes.

In summary, we believe that IVIg binds B cells by interacting with CD22. This ligation interferes with several BCR-mediated signaling pathways. These include inhibition of the PLC $\gamma 2$ cascade, sustained activation of Erk1/2, enhanced p38 activation and down-regulation of PI3K. These changes are associated with the 
induction of CDK inhibitor p27/Kip1, which inhibits cell cycle progression at the $G_{1}$ phase and results in with apoptosis. Modulation of the viability of primary B cells and the growth of cancer line B cell provides for new interest in IVIg therapy in the control of B-cell malignancies.

\section{Acknowledgments}

We thank Ms Geneviève Michel and Ms Simone Forest for expert secretarial assistance.

\section{Authorship}

Contribution: J.-F.S. and D.C. performed the experiments; J.-F.S. and Y.R. analyzed data; P.Y and S.H. designed the research and drafted the manuscript; and R.A.M. helped in drafting and editing the paper.

Conflict-of-interest disclosure: The authors declare no competing financial interests.

Correspondence: Professor Pierre Youinou, Laboratory of Immunology, Brest University Medical School Hospital, BP 824, F29609, Brest, France; e-mail: youinou@univ-brest.fr.

\section{References}

1. Nimmerjahn F, Ravetch JV. Anti-inflammatory actions of IVIg. Annu Rev Immunol. 2008;26:513533.

2. Seite JF, Shoenfeld Y, Youinou P, Hillion S. What is the contents of the magic draft IVIg? Autoimmun Rev. 2008;7(6):435-439.

3. Amran D, Renz H, Lack G, Bradley K, Gelfand EW. Suppression of cytokine-dependent human T-cell proliferation by IVIg. Clin Immunol Immunopathol. 1994;73(2):180-186.

4. Prasad NK, Papoff G, Zeuner A, et al. Therapeutic preparations of normal polyspecific $\lg \mathrm{G}$ (IVIg) induce apoptosis in human lymphocytes and monocytes: a novel mechanism of action of IVIg involving the Fas apoptotic pathway. J Immunol. 1998;161(7):3781-3790.

5. Goodnow CC. Balancing immunity and tolerance: deleting and tuning lymphocyte repertoires. Proc Natl Acad Sci U S A. 1996;93(6):2264-2271.

6. DeFranco AL, Richards JD, Blum JH, et al. Signal transduction by the BCR. Ann N Y Acad Sci. 1995;766:195-201.

7. Rathmell JC. B-cell homeostasis: digital survival or analog growth? Immunol Rev. 2004;197:116128.

8. Tobón GJ, Alard JE, Youinou P, Jamin C. Are au toantibodies triggering endothelial cell apoptosis really pathogenic? Autoimmun Rev. 2009;8(7): 605-610.

9. Dal Porto JM, Gauld SB, Merrell KT, Mills D, Pugh-Bernard AE, Cambier J. BCR signaling 101. Mol Immunol. 2004;41(6-7):599-613.

10. Schulte RJ, Campbell MA, Fischer WH, Sefton BM. Tyrosine phosphorylation of CD22 during B cell activation. Science. 1992;258(5084):10011004.

11. O'Reilly MK, Collins BE, Han S, et al. Bifunctional CD22 ligands use multimeric immunoglobulins as protein scaffolds in assembly of immune complexes on B cells. J Am Chem Soc. 2008;130(24): 7736-7745.

12. Poe JC, Fujimoto $Y$, Hasegawa M, et al. CD22 regulates $\mathrm{B}$ lymphocyte function in vivo through both ligand-dependent and ligand-independent mechanisms. Nat Immunol. 2004;5(10):10781087.

13. Kelm S, Gerlach J, Brossmer R, Danzer CP, Nitschke L. The ligand-binding domain of CD22 is needed for inhibition of the BCR signal, as demonstrated by a novel human CD22-specific inhibitor compound. J Exp Med. 2002;195(9):12071213.

14. Jin L, McLean PA, Neel BG, Wortis HH. Sialic acid binding domains of CD22 are required for negative regulation of BCR signaling. $J$ Exp Med. 2002;195(9):1199-1205.

15. Chiu CW, Dalton M, Ishiai M, Kurosaki T, Chan
AC. BLNK: molecular scaffolding through 'cis'mediated organization of signaling proteins. EMBO J. 2002;21(23):6461-6472.

16. Courtney AH, Puffer EB, Pontrello JK, Yang ZQ, Kiessling LL. Sialylated multivalent antigens engage CD22 in trans and inhibit B cell activation. Proc Natl Acad Sci U S A. 2009;106(8):25002505.

17. Irish JM, Czerwinski DK, Nolan GP, Levy R. Kinetics of BCR signaling in human $B$ cell subsets mapped by phosphospecific flow cytometry. $\mathrm{J} \mathrm{Im}$ munol. 2006;177(3):1581-1589.

18. Nevins JR, Leone G, DeGregori J, Jakoi L. Role of the $\mathrm{Rb} / \mathrm{E} 2 \mathrm{~F}$ pathway in cell growth control. J Cell Physiol. 1997;173(2):233-236.

19. Hillion S, Dueymes M, Youinou P, Jamin C. IL-6 contributes to the expression of RAGs in human mature B cells. J Immunol. 2007;179(10):67906798.

20. Krek W, Ewen ME, Shirodkar S, et al. Negative regulation of the growth-promoting transcription factor E2F-1 by a stably bound cyclin A-dependent protein kinase. Cell. 1994;78(1):161-172.

21. Dussault N, Ducas E, Racine C, et al. Immunomodulation of human $B$ cells following treatment with intravenous immunoglobulins involves increased phosphorylation of extracellular signalregulated kinases 1 and 2. Int Immunol. 2008; 20(11):1369-1379.

22. de Grandmont MJ, Racine C, Roy A, Lemieux R, Néron S. IVIg induces the in vitro differentiation of human $B$ lymphocytes and the secretion of IgG. Blood. 2003;101(8):3065-3073.

23. Proulx DP, Aubin E, Lemieux R, Bazin R. Spontaneous internalization of IVIg in activated $B$ cells. Immunol Lett. 2009;124(1):18-26.

24. Gross AJ, Lyandres JR, Panigrahi AK, Prak ET, DeFranco AL. Developmental acquisition of the Lyn-CD22-SHP-1 inhibitory pathway promotes B cell tolerance. J Immunol. 2009;182(9):53825392.

25. Durandy A, Kaveri SV, Kuijpers TW, et al. Intravenous immunoglobulins-understanding properties and mechanisms. Clin Exp Immunol. 2009; 158(Suppl1):2-13.

26. Duong BH, Tian H, Ota T, et al. Decoration of T-independent antigen with ligands for CD22 and Siglec-G can suppress immunity and induce B cell tolerance in vivo. J Exp Med. 2010;207(1): 173-187.

27. Tuscano J, Engel P, Tedder TF, Kehrl JH. CD22 cross-linking generates BCR-independent signals that activate the JNK/SAPK signaling cascade. Blood. 1999;94(4):1382-1392.

28. Qu Z, Goldenberg DM, Cardillo TM, Shi V, Hansen HJ, Chang CH. Bispecific anti-CD20/22 antibodies inhibit $\mathrm{B}$-cell lymphoma proliferation by a unique mechanism of action. Blood. 2008 111(4):2211-2219.

29. Decker T, Oelsner M, Kreitman RJ, et al. Induction of caspase-dependent programmed cell death in B-cell CLL by anti-CD22 immunotoxins. Blood. 2004;103(7):2718-2726.

30. Bayry J, Bansal K, Kazatchkine MD, Kaveri SV. DC-SIGN and alpha2,6-sialylated IgG Fc interaction is dispensable for the anti-inflammatory activity of IVIg on human dendritic cells. Proc Natl Acad Sci U S A. 2009;106(9):E24.

31. Richards S, Watanabe C, Santos L, Craxton A, Clark EA. Regulation of B-cell entry into the cell cycle. Immunol Rev. 2008;224:183-200.

32. Opferman JT. Apoptosis in the development of the immune system. Cell Death Differ. 2008;15(2): 234-242.

33. He J, Takano T, Ding $\mathrm{J}$, et al. Syk is required for p38 activation and G2/M arrest in B cells exposed to oxidative stress. Antioxid Redox Signal. 2002; 4(3):509-415.

34. Rosini P, De Chiara G, Lucibello M, Garaci E, Cozzolino F, Torcia M. NGF withdrawal induces apoptosis in CESS B cell line through p38 MAPK activation and $\mathrm{Bcl}-2$ phosphorylation. Biochem Biophys Res Commun. 2000;278(3):753-759.

35. Raingeaud J, Gupta S, Rogers JS, et al. Proinflammatory cytokines and environmental stress cause p38 MAP kinase activation by dual phosphorylation on tyrosine and threonine. $J \mathrm{BiO}$ Chem. 1995;270(13):7420-7426.

36. Kim BS, Yoon $\mathrm{KH}$, Oh $\mathrm{HM}$, et al. Involvement of p38 MAP kinase during iron chelator-mediated apoptotic cell death. Cell Immunol. 2002;220(2): 96-106

37. Hsu SC, Gavrilin MA, Tsai MH, Han J, Lai MZ. p38 mitogen-activated protein kinase is involved in Fas ligand expression. J Biol Chem. 1999; 274(36):25769-25776.

38. O'Donnell RT, Pearson D, McKnight HC, Ma YP Tuscano JM. Phosphatase inhibition augments anti-CD22-mediated signaling and cytotoxicity in non-hodgkin's lymphoma cells. Leuk Res. 2009; 33(7):964-969.

39. Lloyd AC. Distinct functions for Erks? J Biol. 2006;5(5):13.

40. Vantaggiato $C$, Formentini I, Bondanza A, Bonini C, Naldini L, Brambilla R. Erk1/2 MPA kinases affect Ras-dependent cell signaling differentially. J Biol. 2006;5(5):14.

41. Herrera L, Farah RA, Pellegrini VA, et al. Immunotoxins against CD19 and CD22 are effective in killing precursor-B acute lymphoblastic leukemia cells in vitro. Leukemia. 2000;14(5):853-858.

42. Deshpande A, Sicinski P, Hinds PW. Cyclins and CDKs in development and cancer: a perspective. Oncogene. 2005;24(17):2909-2915. 